\title{
A CANDIDATE DETECTION OF THE FIRST HYDROSTATIC CORE
}

\author{
Melissa L. Enoch ${ }^{1,5}$, Jeong-Eun LeE ${ }^{2}$, Paul Harver ${ }^{3}$, Michael M. Dunham $^{3}$, and Scott SchneE ${ }^{4}$ \\ ${ }^{1}$ Department of Astronomy, University of California at Berkeley, 601 Campbell Hall, Berkeley, CA 94720, USA; menoch@ berkeley.edu \\ ${ }^{2}$ Department of Astronomy and Space Science, Astrophysical Research Center for the Structure and Evolution of the Cosmos, Sejong University, Seoul 143-747, \\ Republic of Korea \\ ${ }^{3}$ Department of Astronomy, The University of Texas at Austin, 1 University Station, C1400, Austin, TX 78712, USA \\ ${ }^{4}$ National Research Council Canada, Herzberg Institute of Astrophysics, 5071 West Saanich Road, Victoria BC V9E 2E7, Canada \\ Received 2010 July 26; accepted 2010 September 2; published 2010 September 16
}

\begin{abstract}
The first hydrostatic core (FHSC) represents a very early phase in the low-mass star formation process, after collapse of the parent core has begun but before a true protostar has formed. This large (few AU), cool (100 K), pressure-supported core of molecular hydrogen is expected from theory, but has yet to be observationally verified. Here, we present observations of an excellent candidate for the FHSC phase: Per-Bolo 58, a dense core in Perseus that was previously believed to be starless. The $70 \mu \mathrm{m}$ flux of $65 \mathrm{mJy}$, from new deep Spitzer MIPS observations, is consistent with that expected for the FHSC. A low signal-to-noise detection at $24 \mu \mathrm{m}$ leaves open the possibility that Per-Bolo 58 could be a very low luminosity protostar, however. We utilize radiative transfer models to determine the best-fitting FHSC and protostar models to the spectral energy distribution and $2.9 \mathrm{~mm}$ visibilities of Per-Bolo 58. The source is consistent with an FHSC with some source of lower opacity through the envelope allowing $24 \mu \mathrm{m}$ emission to escape; a small outflow cavity and a cavity in the envelope are both possible. While we are unable to rule out the presence of a protostar, if present it would be one of the lowest luminosity protostellar objects yet observed, with an internal luminosity of $\sim 0.01 L_{\odot}$.
\end{abstract}

Key words: infrared: ISM - radiative transfer - stars: formation - submillimeter: ISM

Online-only material: color figures

\section{INTRODUCTION}

One stage of the low-mass star formation paradigm has yet to be observed: the first core or first hydrostatic core (FHSC). During this brief phase between the initiation of core collapse and the appearance of a "true" protostar, the growing central core of molecular hydrogen $\left(\mathrm{H}_{2}\right)$ is heated to the point at which it obtains hydrostatic balance. When the central temperature of the first core reaches approximately $2000 \mathrm{~K}$, the $\mathrm{H}_{2}$ is dissociated and a second collapse forms the true protostar.

The FHSC has long been expected from theory (Larson 1969), but has not yet been observationally verified. Chen et al. (2010) recently presented a promising candidate, L1448 IRS2E, based on detection of a $\mathrm{CO}$ outflow without a corresponding midinfrared source. The observed outflow is faster than would be expected for a typical FHSC (Machida et al. 2008), but might be consistent with the very end of the FHSC phase.

The expected effective temperature of the FHSC, which has a radius of several $\mathrm{AU}$, is approximately $100 \mathrm{~K}$; as the majority of this cool emission is absorbed and re-radiated by the even cooler envelope, the emergent spectrum of the FHSC is very similar to that of a starless core. The strongest observational signature is likely to be an increase in luminosity for $\lambda \sim 40-100 \mu \mathrm{m}$ as compared to starless cores (Boss \& Yorke 1995). Furthermore, given the expected short lifetime of the FHSC $\left(t \sim 10^{3}-3 \times\right.$ $10^{4}$ yr; Omukai 2007; Boss \& Yorke 1995), there should only be a few observable even in large samples of cores and protostars. Based on the relative lifetimes of the FHSC and true protostars $\left(5.4 \times 10^{5} \mathrm{yr}\right.$; Evans et al. 2009), we expect only one FHSC for every $18-540$ protostars. Before large Spitzer surveys such as the "From Molecular Cores to Planet-forming Disks" Legacy Program ("Cores to Disks" or c2d; Evans et al. 2003), the

\footnotetext{
5 Spitzer Fellow.
}

number of known protostars in any given region was typically too low to expect even one FHSC.

We have recently completed a census of starless cores and embedded protostars in the Perseus, Serpens, and Ophiuchus molecular clouds (Enoch et al. 2008, 2009b), based on largescale Bolocam $1.1 \mathrm{~mm}$ continuum surveys (Enoch et al. 2006, 2007; Young et al. 2006) and Spitzer c2d surveys (Jørgensen et al. 2006; Harvey et al. 2006, 2007; Rebull et al. 2007; Padgett et al. 2008). Perseus, Serpens, and Ophiuchus each harbor between 30 and 70 embedded protostars-objects after the second collapse, and thus more evolved than FHSCs, but still embedded in and accreting from their natal cores-and should contain at least a few FHSCs (if the lifetime is longer than $10^{4}$ yr). Spitzer was sufficiently sensitive to detect $70 \mu \mathrm{m}$ emission from a $0.015 M_{\odot}$ FHSC at a distance of $250 \mathrm{pc}$ (Omukai 2007), but large surveys such as c2d were not deep enough at $70 \mu \mathrm{m}$ to detect the FHSC.

In this Letter, we present new deep Multiband Imaging Photometer for Spitzer (MIPS) observations that reveal a candidate FHSC in the Perseus molecular cloud. Utilizing literature data, we compare the spectral energy distribution (SED) and millimeter visibilities of this source to radiative transfer models of an FHSC and very low luminosity protostar.

\section{TARGET SELECTION}

Figure 1 shows the expected observed SED of an FHSC, from the radiative transfer models of Young \& Evans (2005). Based on these models, an unambiguous detection of the FHSC requires detection of compact $70 \mu \mathrm{m}$ emission in a dense core with no emission at $\lambda \lesssim 24 \mu \mathrm{m}$. The $70 \mu \mathrm{m}$ sensitivity limit of the c2d survey was approximately $75 \mathrm{mJy}(5 \sigma)$, somewhat higher than the flux expected for a typical FHSC (Boss \& Yorke 1995; Young \& Evans 2005). Thus, any FHSCs appeared as starless 


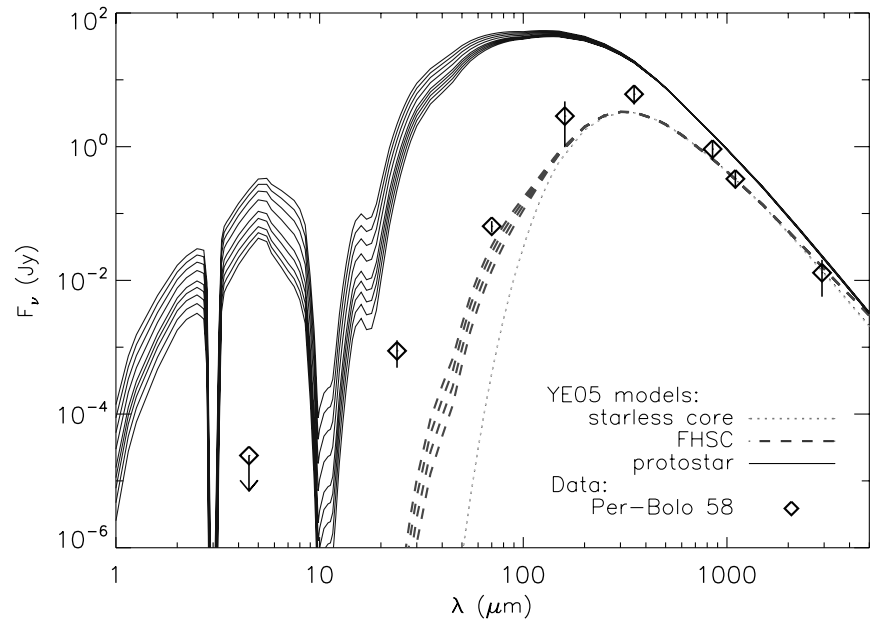

Figure 1. Radiative transfer models from Young \& Evans (2005), for a $1 M_{\odot}$ core at the distance of Perseus $(d=250 \mathrm{pc})$. SEDs represent a time sequence, from a starless core (dotted line) to FHSC (dashed lines) to true protostar (solid lines), with short-wavelength flux increasing monotonically with time and time steps of 2000 yr. The observed SED of Per-Bolo 58 (diamonds; see Section 3) includes fluxes from Table 1 . The $70 \mu \mathrm{m}$ flux of Per-Bolo 58 is roughly consistent with that expected for the FHSC. The $24 \mu \mathrm{m}$ detection is inconsistent with these simple spherical models of the FHSC, however.

cores in our census, and deeper $70 \mu \mathrm{m}$ follow-up observations were necessary to reveal them. ${ }^{6}$

We selected targets from our census of starless cores (Enoch et al. 2008), which includes 108 cores and is complete to $M_{\text {core }} \gtrsim 0.2 M_{\odot}$, based on three criteria. (1) Visual inspection of the c2d $70 \mu \mathrm{m}$ maps at starless core positions, looking for faint emission below the formal $5 \sigma$ detection limit. (2)

6 We did an initial check of the c2d catalogs for starless cores detected only at $70 \mu \mathrm{m}$ but found no such objects.
Starless cores with mass-to-size ratios above the critical value for stable Bonnor-Ebert spheres (central to surface density ratio $\rho_{c} / \rho_{0}>14$, or $M\left(M_{\odot}\right) / \mathrm{FWHM}(\mathrm{pc})>31.9$ for $T=$ $10 \mathrm{~K})$. These cores are likely to be collapsing, but the lack of near-infrared or mid-infrared emission indicates that they have not yet formed a true protostar. (3) Spatially compact starless cores, either unresolved by the $31^{\prime \prime}$ Bolocam beam or compact in follow-up CARMA 2.9 mm interferometric maps (M. L. Enoch et al. 2010, in preparation). Again, these cores have high central density and may be collapsing.

In the three clouds, seven starless cores met one or more of the above criteria. This is by no means a complete or unbiased sample; rather, we used these criteria to identify interesting objects for deeper follow-up observations.

\section{OBSERVATIONS AND RESULTS}

Deep $70 \mu \mathrm{m}$ maps of our seven targets were obtained with the MIPS on the Spitzer Space Telescope (Werner et al. 2004) during 2008 October 20-27. Integration times ranged between 1100 and $2000 \mathrm{~s} \mathrm{pixel}^{-1}$, depending on the brightness of the background estimated from c $2 \mathrm{~d}$ maps. The resulting $5 \sigma$ detection limit of $\sim 7.5 \mathrm{mJy}$ was chosen based on the models of Omukai (2007), to detect FHSCs with mass $\gtrsim 0.015 M_{\odot}$ at the distance of Perseus and Serpens (250-260 pc).

Data were reduced using the c2d pipeline (Evans et al. 2007). One source was clearly detected at $70 \mu \mathrm{m}$ : [EYG2006] Bolo 58 (R.A. = 0329 25.7, decl. $=+3128$ 16.3), hereafter Per-Bolo 58, a dense core identified as starless by both Enoch et al. (2006) and Hatchell et al. (2007). Per-Bolo 58 was included in our target list based on faint emission in the $\mathrm{c} 2 \mathrm{~d} 70 \mu \mathrm{m}$ map and a spatially compact $1.1 \mathrm{~mm}$ core. It is located in the outskirts of the NGC 1333 young cluster (see Figure 2, right).

The $70 \mu \mathrm{m}$ flux of Per-Bolo 58 is $65 \pm 6 \mathrm{mJy}$. A subsequent search of the c2d deep catalogs (Evans et al. 2007) revealed

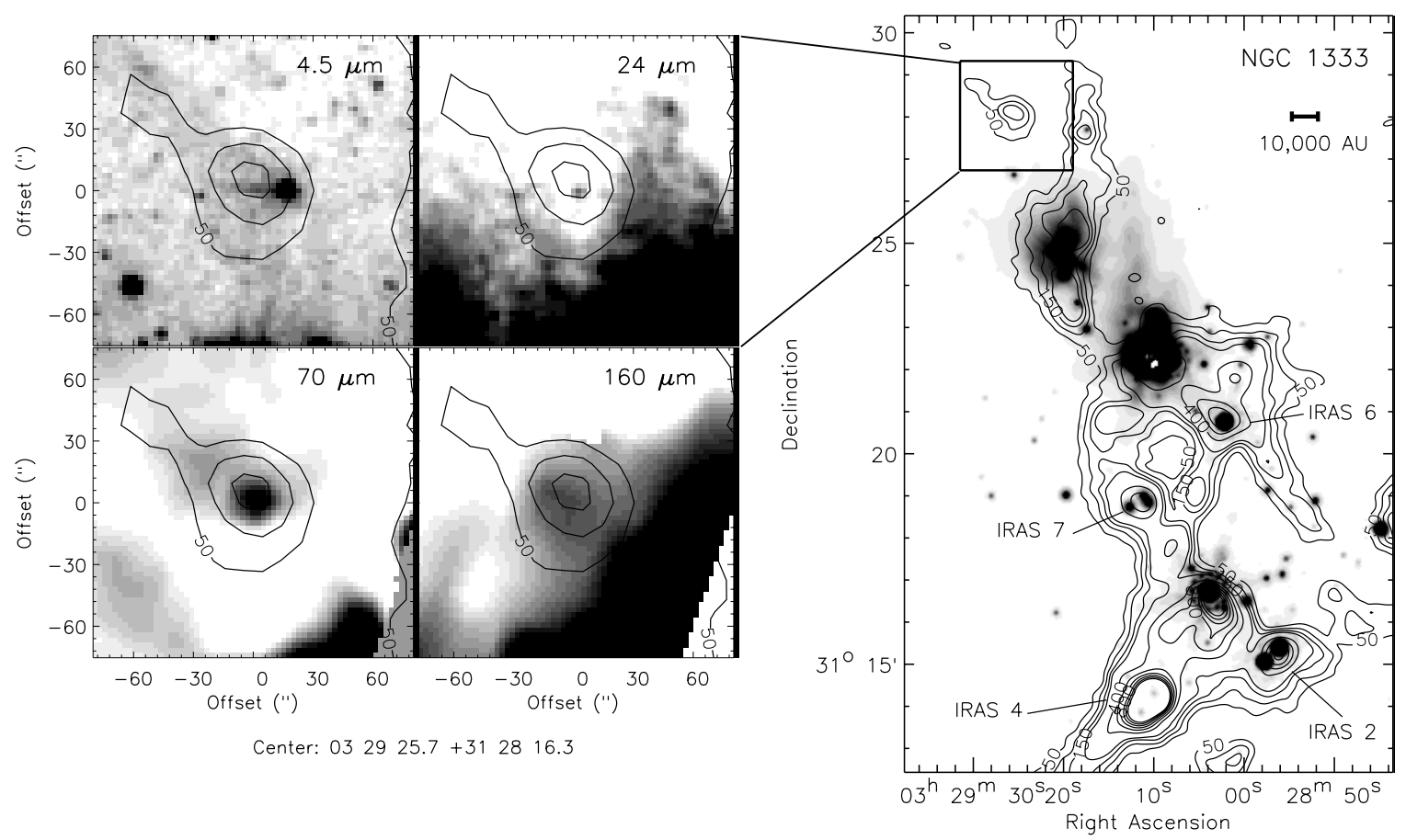

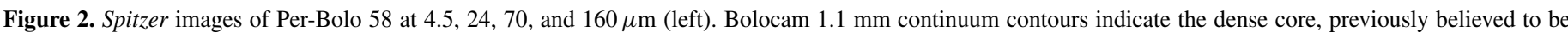

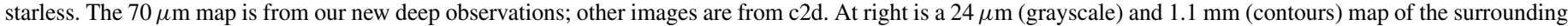

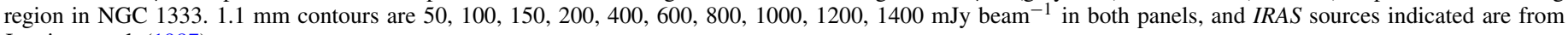
Jennings et al. (1987). 

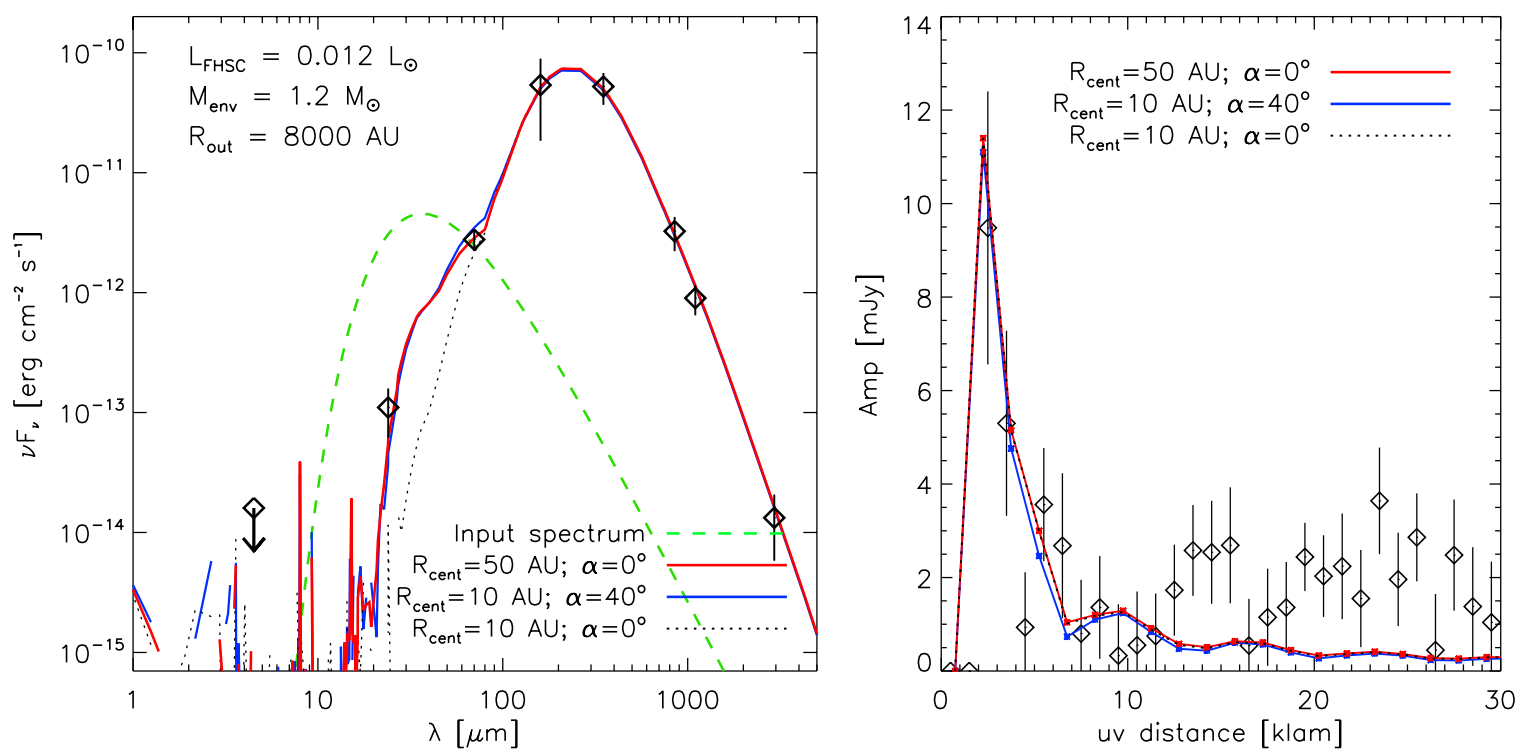

Figure 3. Best-fitting FHSC model compared to the observed SED (left) and CARMA 2.9 mm visibilities (right; Schnee et al. 2010) of Per-Bolo 58. While an FHSC provides a good fit to the SED, either a cavity in the inner envelope $\left(R_{\text {cent }} \sim 50 \mathrm{AU}\right)$, or outflow $(\alpha \gtrsim 20 \mathrm{deg})$ is required to match the $24 \mu \mathrm{m}$ flux. The two best models, with $R_{\text {cent }}=50 \mathrm{AU}, \alpha=0 \mathrm{deg}$ (red) and $R_{\text {cent }}=10 \mathrm{AU}, \alpha=40 \mathrm{deg}$ (blue) are nearly indistinguishable. The input FHSC spectrum $\left(L=0.012 L_{\odot}, R=\right.$ $1.7 \mathrm{AU}, T_{\mathrm{eff}}=100 \mathrm{~K}$; dashed line), and a model with no cavity (dotted line) are also shown for comparison. The millimeter visibilities (right) help to constrain the envelope outer radius, with a best fit for $R_{\text {out }}=8000 \mathrm{AU}$.

(A color version of this figure is available in the online journal.)

Table 1

Observed SED of Per-Bolo 58

\begin{tabular}{lcccc}
\hline \hline $\begin{array}{l}\text { Wavelength } \\
(\mu \mathrm{m})\end{array}$ & $\begin{array}{c}\text { Flux } \\
(\mathrm{mJy})\end{array}$ & $\begin{array}{c}\sigma(\text { Flux }) \\
(\mathrm{mJy})\end{array}$ & $\begin{array}{c}\text { Aperture } \\
\text { Diameter }\left({ }^{\prime \prime}\right)\end{array}$ & Notes \\
\hline 4.5 & 0.024 & 0.07 & 2.2 & Upper limit; Spitzer $(\mathrm{c} 2 \mathrm{~d})$ \\
24 & 0.88 & 0.24 & 7 & Spitzer $(\mathrm{c} 2 \mathrm{~d})$ \\
70 & 65 & 6 & 17 & Spitzer; this work \\
160 & 2870 & 1600 & 40 & Spitzer (c2d) \\
350 & 6100 & 1200 & 40 & SHARC II; M. M. Dunham et al. 2010, in preparation \\
850 & 920 & 200 & 18 & SCUBA; Hatchell et al. (2005) \\
1100 & 330 & 30 & 40 & Bolocam; Enoch et al. (2006) \\
2930 & 13 & 6 & 15 & CARMA; Schnee et al. (2010) \\
\hline
\end{tabular}

Notes. Continuum fluxes are calculated by aperture photometry within the aperture listed, with the exception of the $2.9 \mathrm{~mm}$ flux, which is determined by a Gaussian fit $\left(\mathrm{FWHM}=15^{\prime \prime}\right)$. Our $2.9 \mathrm{~mm}$ flux does not include the Sunyaev-Zel'dovich Array data from Schnee et al. (2010), which probes larger spatial scales than we include in our models.

a low signal-to-noise $24 \mu \mathrm{m}$ detection at the position of PerBolo 58, with a flux of $0.88 \pm 0.24 \mathrm{mJy}$. This source was not included in the "high reliability" or "young stellar object candidate" c $2 \mathrm{~d}$ catalogs, as it did not meet the $7 \sigma$ signal-tonoise requirement. Given the good positional correspondence and point-like emission, we believe that the $24 \mu \mathrm{m}$ source is associated with Per-Bolo 58. There is also a nearby low signalto-noise $4.5 \mu \mathrm{m}$ detection $(0.024 \pm 0.007 \mathrm{mJy})$ that we do not believe is associated, as the only point-like emission is offset by $10^{\prime \prime}$ from the $24 \mu \mathrm{m}$ and $70 \mu \mathrm{m}$ sources. We treat the $4.5 \mu \mathrm{m}$ point as an upper limit. We estimate a $160 \mu \mathrm{m}$ flux of $2.8 \mathrm{Jy}$ from the c2d MIPS $160 \mu \mathrm{m}$ map; this value has a large uncertainty ( $\sim 50 \%)$ due to non-uniform extended emission in NGC 1333. Spitzer images at 4.5, 24, 70, and $160 \mu \mathrm{m}$ are shown in Figure 2.

All known continuum fluxes for Per-Bolo 58 are given in Table 1, and the observed SED is shown in Figure 1. In addition to the Spitzer data described above, we include SHARC II $350 \mu \mathrm{m}$ (M. M. Dunham et al. 2010, in preparation), SCUBA $850 \mu \mathrm{m}$ (Hatchell et al. 2005), and Bolocam $1.1 \mathrm{~mm}$ (Enoch et al. 2006) fluxes as well as the CARMA $2.9 \mathrm{~mm}$ map from
Schnee et al. (2010). The interferometric CARMA $2.9 \mathrm{~mm}$ map provides both a long wavelength flux measurement and a measure of the resolved radial intensity profile. The $2.9 \mathrm{~mm}$ visibility amplitudes versus $u v$-distance are shown in Figures 3 and 4.

\section{DISCUSSION}

Our $70 \mu \mathrm{m}$ detection of Per-Bolo 58 indicates the presence of an internal luminosity source in this dense core, with a flux consistent with that expected for an FHSC. The detection at $24 \mu \mathrm{m}$ is inconsistent with an FHSC surrounded by a spherically symmetric envelope, however (Figure 1), leaving open the possibility that Per-Bolo 58 may be a very low luminosity protostar. We discuss these possibilities below.

\subsection{Radiative Transfer Models}

We use RADMC, a two-dimensional Monte Carlo radiative transfer code (Dullemond \& Dominik 2004) to model an FHSC and low-luminosity protostar. We adopt a density profile that 
includes a rotating infalling envelope characterized by total mass $M_{\text {env }}$, centrifugal radius $R_{\text {cent }}$, and outer radius $R_{\text {out }}$ (e.g., Ulrich et al. 1967), and outflow cavity characterized by full opening angle $\alpha$. The envelope mass is held fixed at $M_{\mathrm{env}}=1.2 M_{\odot}$, as calculated from the $1.1 \mathrm{~mm}$ flux (see Enoch et al. 2006) assuming a dust temperature of $T=8 \mathrm{~K}$, consistent with radiative transfer models of such a low-luminosity source. Details of the density profile and modeling are as in Enoch et al. (2009a).

Dust opacities are from Table 1, Column 5 of Ossenkopf \& Henning (1994) for dust grains with thin ice mantles, including scattering. Following Dunham et al. (2010), we remove the effects of excess backscattering of the interstellar radiation field (ISRF) by subtracting the flux for a starless core model at short wavelengths. Dunham et al. (2010) make this correction for $\lambda \leqslant 10 \mu \mathrm{m}$; here, we additionally include the small plateau of ISRF emission at $\lambda=10-40 \mu \mathrm{m}$. While this correction affects the shape of our model SEDs at short wavelengths, the correction at $24 \mu \mathrm{m}$ is small, so it does not significantly affect the model fits.

To match the peak of the SED at $80 \mu \mathrm{m}<\lambda<1000 \mu \mathrm{m}$, we include an ISRF that is a factor of 3 stronger than the "Black-Draine" ISRF (Evans et al. 2001; Black 1994; Draine 1978). A more intense radiation field may be expected in NGC 1333, where there is significant extended emission at $\lambda \leqslant 24 \mu \mathrm{m}$ (e.g., Figure 2). Increasing the ISRF improves the overall fit of all models, but does not change the best-fitting envelope parameters, because the SED peak depends primarily on fixed parameters $\left(M_{\mathrm{env}}, \mathrm{ISRF}\right)$.

\subsection{Per-Bolo 58 as a First Hydrostatic Core}

Our FHSC models include a cool $\left(T_{\text {eff }}=100 \mathrm{~K}\right)$, large ( $R \sim 2 \mathrm{AU}$, where the actual radius is determined from $L_{\text {int }}=4 \pi R^{2} \sigma T_{\text {eff }}{ }^{4}$ ) internal luminosity source. We run a grid of models varying $L_{\text {int }}\left(0.006,0.012,0.025 L_{\odot}\right), R_{\text {out }}(4000,6000$, $8000,10000 \mathrm{AU}), R_{\text {cent }}(10,50,100,200,300 \mathrm{AU}), \alpha(0,5,10$, $20,40,60 \mathrm{deg})$, and inclination $(i ; 5,10,15 \ldots 90 \mathrm{deg})$.

Models are compared to the observed SED of Per-Bolo 58 using a $\chi^{2}$ analysis to constrain $L_{\text {int }}, R_{\text {cent }}, \alpha$, and $i$. An internal luminosity of $0.012 L_{\odot}$ is clearly preferred by the SED, and we derive other parameters with $L_{\text {int }}$ fixed. The SED is rather insensitive to $R_{\text {out }}$, so we also compute the visibility amplitudes of each model as a function of $u v$-distance and compare to the $2.9 \mathrm{~mm}$ CARMA observations from Schnee et al. (2010). At small $u v$-distances $(\leqslant 10 k \lambda)$, the visibilities are sensitive to the spatial extent of the envelope (see Enoch et al. 2009a for more details), and we find a best fit for $R_{\text {out }}=8000$ AU.

The best-fitting FHSC model $\left(L_{\text {int }}=0.012 L_{\odot}, T_{\text {eff }}=\right.$ $100 \mathrm{~K}, R=1.7 \mathrm{AU}, R_{\text {cent }}=50 \mathrm{AU}, R_{\text {out }}=8000 \mathrm{AU}$, $\alpha=0 \mathrm{deg}$, and $i=25 \mathrm{deg}$ ) is compared to the observed SED and $2.9 \mathrm{~mm}$ visibilities in Figure 3. Another well-fitting model with $R_{\text {cent }}=10 \mathrm{AU}$ and $\alpha=40 \mathrm{deg}$ is also shown. A number of models have similar $\chi^{2}$ values, but all have either an envelope cavity $\left(R_{\text {cent }} \geqslant 50\right.$ AU) or an outflow ( $\left.\alpha \geqslant 20 \mathrm{deg}\right)$. Models without an envelope cavity are clearly unable to match the observed $24 \mu \mathrm{m}$ point, due to the high opacity through the envelope; such a model with $R_{\text {cent }}=10 \mathrm{AU}$ and $\alpha=0$ deg is shown in Figure 3 for comparison.

Although an envelope cavity is not expected from simple physical models of the FHSC, in which the envelope extends down to the FHSC and no outflow is present, there are several cases in which we might expect a cavity. MHD calculations have shown that the FHSC can drive a molecular outflow (e.g.,
Machida et al. 2008). Such early outflows should be weak, but could evacuate enough envelope material to allow a small amount of $24 \mu \mathrm{m}$ flux to escape. Similarly, a larger centrifugal radius might be expected if the inner envelope were cleared by a binary source or weak outflow. We note that $4.5 \mu \mathrm{m}$ is the Spitzer band associated with shock emission in outflows, and the nearby weak $4.5 \mu \mathrm{m}$ emission could be tracing a weak outflow.

\subsection{Per-Bolo 58 as a Very Low Luminosity Protostar}

A number of dense cores previously believed to be starless were found by Spitzer to harbor very low luminosity objects (VeLLOs; e.g., Young et al. 2004). The best current explanation of VeLLOs is that they are true protostars, but with low internal luminosities due either to very low masses or very low accretion rates. Most VeLLOs have internal luminosities between 0.02 and $0.1 L_{\odot}$ (Dunham et al. 2008); although the 24 and $70 \mu \mathrm{m}$ fluxes of Per-Bolo 58 are lower than typical VeLLOs, it may be an extreme example of a low-luminosity protostellar population. At least two other protostellar sources with similar luminosities are known: a candidate embedded proto-brown dwarf in Taurus with $L_{\text {int }} \sim 0.003 L_{\odot}($ SSTB213 J041757; Barrado et al. 2009) and Cha-MMS 1, which has an estimated internal luminosity of 0.01-0.02 $L_{\odot}$ (Belloche et al. 2006). ${ }^{7}$

To model Per-Bolo 58 as a protostar, we use a similar grid as above, but include a hot internal luminosity source typical of a true protostar $\left(T_{\mathrm{eff}}=3000 \mathrm{~K}, R \sim 1 R_{\odot}\right)$. The best-fitting protostar model $\left(L_{\text {int }}=0.012 L_{\odot}, R_{\text {cent }}=50 \mathrm{AU}, R_{\text {out }}=\right.$ $8000 \mathrm{AU}, \alpha=10 \mathrm{deg}$, and $i=30 \mathrm{deg}$ ) is shown in Figure 4. Protostar models with relatively high inclinations $(i>\alpha)$ are nearly indistinguishable from the corresponding FHSC models; in both cases essentially all of the emission from the central source is reprocessed by the envelope, masking the details of the internal luminosity source. We can rule out protostar models with low inclinations, however, which produce far too much emission in the near-infrared.

Again, an internal luminosity of $0.012 L_{\odot}$ is strongly favored. This value is corroborated by the relationship between internal luminosity and observed $70 \mu \mathrm{m}$ flux determined by Dunham et al. (2008): $L_{\text {int }}=3.3 \times 10^{8} F_{70}^{0.94} L_{\odot}$, where $F_{70}$ is the flux (in erg cm$~^{-2} \mathrm{~s}^{-1}$ ) at $140 \mathrm{pc}$. For Per-Bolo 58 this relationship yields $L_{\text {int }}=0.014 L_{\odot}$. If Per-Bolo 58 is a true protostar then it is one of the lowest luminosity-embedded protostars known, with $L_{\text {int }} \sim 0.01 L_{\odot}$.

\subsection{Possible Observational Tests}

We are currently unable to conclusively determine the evolutionary state of Per-Bolo 58. While the Herschel Gould Belt survey (André et al. 2010) will further refine the SED, additional observations are needed to distinguish an FHSC with a small envelope cavity from an extremely low luminosity protostar. Possible observational tests include measuring the outflow velocity and finding evidence of water ice evaporation.

Numerical MHD models suggest that the FHSC can drive a molecular outflow, but with quite low velocity $\left(v \sim 3 \mathrm{~km} \mathrm{~s}^{-1}\right.$; Machida et al. 2008; Tomida et al. 2010). As protostellar outflows typically have speeds of $10-20 \mathrm{~km} \mathrm{~s}^{-1}$ (e.g., Arce \& Sargent 2006), detecting an outflow and measuring its velocity, either in the millimeter or with Herschel spectroscopy, could test our hypothesis that Per-Bolo 58 is an FHSC. No outflow was detected in the HARP ${ }^{12} \mathrm{CO}(3-2)$ study of Hatchell \& Dunham

\footnotetext{
7 Belloche et al. (2006) note that Cha-MMS 1 may also be an FHSC candidate, however.
} 

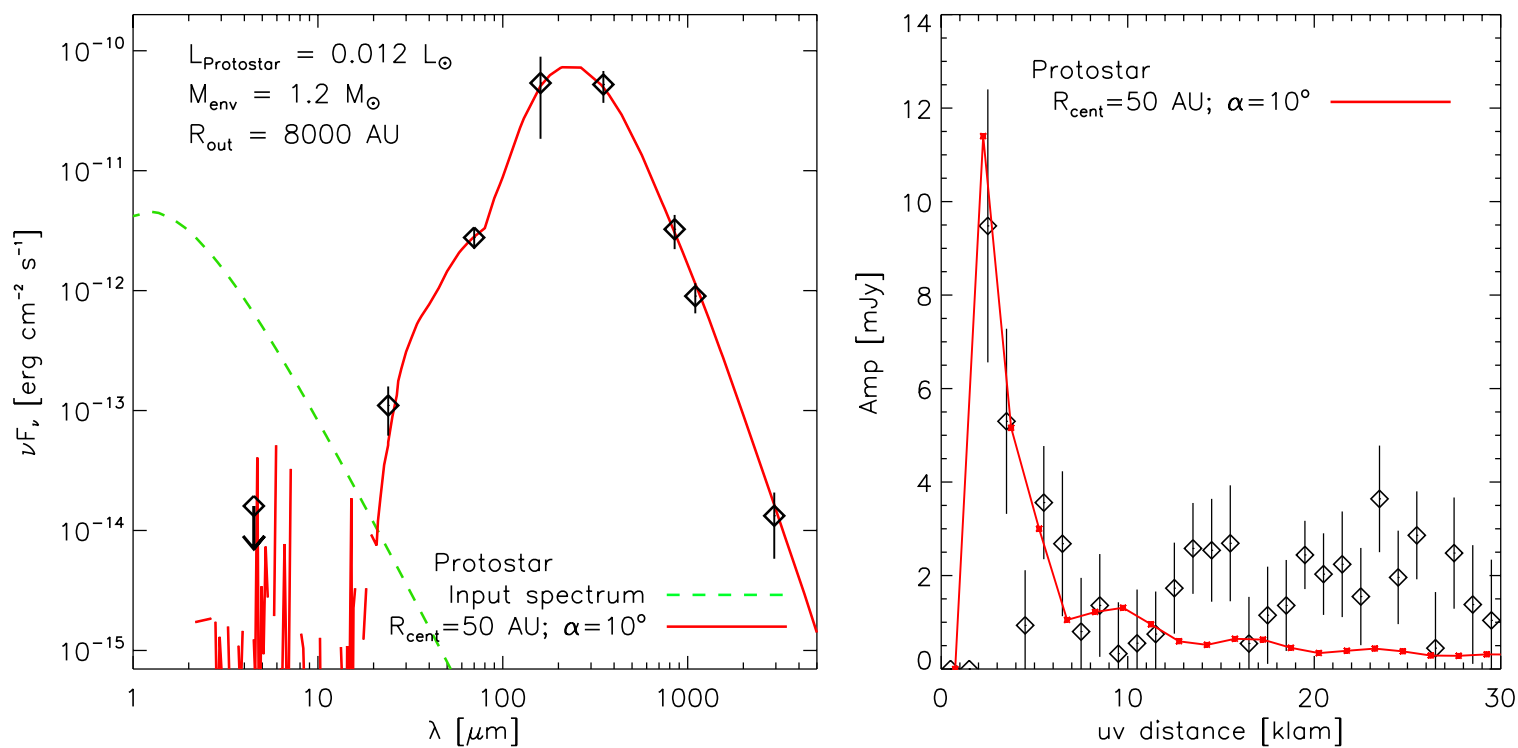

Figure 4. Same as Figure 3, but for the best-fitting protostar model, with $L_{\text {int }}=0.012 L_{\odot}$ and a narrow outflow cavity $(\alpha \sim 10 \mathrm{deg})$. Despite the much hotter input spectrum $(3000 \mathrm{~K})$, the best-fitting protostar SEDs is nearly indistinguishable from the best-fitting FHSC, because most of the internal luminosity is re-processed by the dense envelope. Likewise, the $2.9 \mathrm{~mm}$ visibilities depend only on the envelope density profile, which is similar in both sets of models.

(A color version of this figure is available in the online journal.)

(2009), but an FHSC outflow should be below their detection criteria (line wing strength above $1.5 \mathrm{~K}$ at $3 \mathrm{~km} \mathrm{~s}^{-1}$ from the core velocity).

Unlike the FHSC, for which the maximum dust temperature in the envelope is only $\sim 80 \mathrm{~K}$, temperatures in our protostar models are high enough in the inner few AU (up to $300 \mathrm{~K}$ ) to evaporate water ice (e.g., Fraser et al. 2001). ALMA should be capable of detecting the resulting $\mathrm{H}_{2}^{18} \mathrm{O}$ (Jørgensen \& van Dishoeck 2010) HDO lines, even with the beam dilution of a 0 '.08 (20 AU at $250 \mathrm{pc}$ ) beam. Given the outflow non-detection at the level expected for a typical protostar, finding chemical signatures indicative of high gas temperatures may be the best way to rule out Per-Bolo 58 as an FHSC.

\section{CONCLUSIONS}

In an effort to observationally verify the theoretically predicted FHSC phase, we have obtained deep Spitzer $70 \mu \mathrm{m}$ images of a small sample of dense starless cores. Per-Bolo 58 is detected at the level expected for the FHSC, making it a very promising FHSC candidate. A weak detection at $24 \mu \mathrm{m}$ leaves open the possibility that it might be an extremely low luminosity protostar, however.

We are able to reproduce the observed SED and $2.9 \mathrm{~mm}$ visibilities of Per-Bolo 58 with radiative transfer models of an FHSC, although some source of lower envelope opacity allowing $24 \mu \mathrm{m}$ emission to escape is required: either a small outflow cavity or a spherical cavity in the envelope of $\sim 50 \mathrm{AU}$. While we cannot rule out the possibility that Per-Bolo 58 has already formed a true protostar, with an internal luminosity $L_{\text {int }} \sim 0.01 L_{\odot}$, it would be one of the lowest luminosity protostars known. Additional observational tests to clarify the evolutionary state of Per-Bolo 58 include measuring the velocity of any outflow present and looking for evidence of water ice evaporation in the inner few AU. Finally, we note that if Per-Bolo 58 is a true FHSC, then its observational similarity to VeLLOs (which are defined by luminosity alone) suggests that some VeLLOs currently thought to be protostellar might in fact be members of the FHSC phase.
We thank the anonymous referee for helpful comments, C. Dullemond for the use of RADMC, and N. Evans for many useful discussions. This work is based on observations made with the Spitzer Space Telescope, operated by the Jet Propulsion Laboratory (JPL), California Institute of Technology (Caltech) under a contract with NASA. Support was provided by NASA through an award issued by JPL/Caltech, by the Spitzer Fellowship Program, and by the National Research Foundation of Korea (NRF) grant funded by the Korea government (No. 2009-0062866) and Basic Science Research Program through the NRF funded by the Ministry of Education, Science and Technology (No. 2010-0008704).

\section{REFERENCES}

André, P., et al. 2010, A\&A, 518, 102

Arce, H. G., \& Sargent, A. I. 2006, ApJ, 646, 1070

Barrado, D., et al. 2009, A\&A, 508, 859

Belloche, A., Parise, B., van der Tak, F. F. S., Schilke, P., Leurini, S., Güsten, R., \& Nyman, L.-Å. 2006, A\&A, 454, L51

Black, J. H. 1994, in ASP Conf. Ser. 58, The First Symposium on the Infrared Cirrus and Diffuse Interstellar Clouds, ed. R. M. Cutri \& W. B. Latter (San Francisco, CA: ASP), 355

Boss, A. P., \& Yorke, H. W. 1995, ApJ, 439, L55

Chen, X., Arce, H. G., Zhang, Q., Bourke, T. L., Launhardt, R., Schnalzl, M., \& Henning, T. 2010, ApJ, 715, 1344

Draine, B. T. 1978 , ApJS, 36, 595

Dullemond, C. P., \& Dominik, C. 2004, A\&A, 417, 159

Dunham, M. M., Crapsi, A., Evans, N. J., II, Bourke, T. L., Huard, T. L., Myers, P. C., \& Kauffmann, J. 2008, ApJS, 179, 249

Dunham, M. M., Evans, N. J., Terebey, S., Dullemond, C. P., \& Young, C. H. 2010, ApJ, 710, 470

Enoch, M. L., Corder, S., Dunham, M. M., \& Duchêne, G. 2009a, ApJ, 707, 103

Enoch, M. L., Evans, N. J., II, Sargent, A. I., \& Glenn, J. 2009b, ApJ, 692, 973

Enoch, M. L., Evans, N. J., II, Sargent, A. I., Glenn, J., Rosolowsky, E., \& Myers, P. C. 2008, ApJ, 684, 1240

Enoch, M. L., Glenn, J., Evans, N. J., II, Sargent, A. I., Young, K. E., \& Huard, T. L. 2007, ApJ, 666, 982

Enoch, M. L., et al. 2006, ApJ, 638, 293

Evans, N. J., II, Rawlinge, J. M. C., Shirley, Y. L., \& Mundy, L. G. 2001, ApJ, 557, 193

Evans, N. J., II, et al. 2003, PASP, 115, 965 
Evans, N. J., II, et al. 2007, Final Delivery of Data from the c2d Legacy Project: IRAC and MIPS (Pasadena, CA: SSC)

Evans, N. J., II, et al. 2009, ApJS, 181, 321

Fraser, H. J., Collings, M. P., McCoustra, M. R. S., \& Williams, D. A. 2001, MNRAS, 327, 1165

Harvey, P. M., et al. 2006, ApJ, 644, 307

Harvey, P. M., et al. 2007, ApJ, 663, 1139

Hatchell, J., \& Dunham, M. M. 2009, A\&A, 502, 139

Hatchell, J., Fuller, G. A., Richer, J. S., Harries, T. J., \& Ladd, E. F. 2007, A\&A, 468, 1009

Hatchell, J., Richer, J. S., Fuller, G. A., Qualtrough, C. J., Ladd, E. F., \& Chandler, C. J. 2005, A\&A, 440, 151

Jennings, R. E., Cameron, D. H. M., Cudlip, W., \& Hirst, C. J. 1987, MNRAS, 226, 461

Jørgensen, J. K., \& van Dishoeck, E. F. 2010, ApJ, 710, L72

Jørgensen, J. K., et al. 2006, ApJ, 645, 1246
Larson, R. B. 1969, MNRAS, 145, 271

Machida, M., Inutsuka, S.-I., \& Matsumoto, T. 2008, ApJ, 676, 1088

Omukai, K. 2007, PASJ, 59, 589

Ossenkopf, V., \& Henning, Th. 1994, A\&A, 291, 943

Padgett, D. L., et al. 2008, ApJ, 672, 1013

Rebull, L. M., et al. 2007, ApJS, 171, 447

Schnee, S., Enoch, M., Johnstone, D., Culverhouse, T., Leitch, E., Marrone, D. P., \& Sargent, A. 2010, ApJ, 718, 306

Tomida, K., Tomisaka, K., Matsumoto, T., Ohsuga, K., Machida, M. N., \& Saigo, K. 2010, ApJ, 714, L58

Ulrich, B. T., Neugebauer, G., McCammon, D., Leighton, R. B., Hughes, E. E., \& Becklin, E. 1967, ApJ, 147, 858

Werner, M., et al. 2004, ApJS, 154, 1

Young, C. H., \& Evans, N. J., II. 2005, ApJ, 627, 293

Young, C. H., et al. 2004, ApJS, 154, 396

Young, K. E., et al. 2006, ApJ, 644, 326 